\title{
Frictional effect of spherical convex textured rigid bodies sliding on smooth PDMS
}

\author{
Zixin ZHANG ${ }^{1}$, Zhuangzhuang $\mathrm{CHU}^{1}$ and Xuli ZHU ${ }^{*}$ \\ ${ }^{1}$ College of Mechanical and Electronic Engineering, Shandong University of Science and Technology, Qingdao, Shandong, 266590, \\ China
}

\begin{abstract}
Surface texturing was applied to flexible friction couples to improve their tribological behavior. Spherical convex texture was fabricated on the surface of photosensitive resin by stereolithography (SLA), the friction coefficient between the textured resin sample and smooth polydimethylsiloxane (PDMS) under the conditions of low sliding speed and dry friction was measured using a self-made frictional test bench. It was found that surface texture is capable of reducing friction at low applied normal load compared with the non textured friction couple, as well as increasing friction at high load, in addition, smaller texture radii tend to increase friction. At last, the friction mechanism of the textured flexible friction couple was discussed by establishing a mechanical model.
\end{abstract}

\section{Introduction}

There are many flexible frictional contact phenomena in daily life, such as the contact between animal skin and environment, the contact between car wiper and windshield, the contact between shoe sole and ground. Rubber materials have been widely used in flexible friction couples due to their excellent comprehensive performance, especially in sealing, friction transmission and friction braking. It has been a very important research topic to improve the performance of parts or mechanisms by improving the tribological properties of friction couples [1].

Surface texturing has been considered as an effective method to improve the friction coefficient and wear rate of friction couples [2-4]. Huang et al. [5] experimentally investigated the coupling effects of surface texture and surface wettability on contact lubrication of elastomers, it is found that the friction behavior of the surface texture is quite different under different lubrication conditions. Li et al. [6] tested the effect of friction speed on the friction coefficient between steel bearing ball and textured polydimethylsiloxane (PDMS) disc under water lubrication, and found that the textured sample reduces friction under low sliding speed and increases friction under high sliding speed. On the aspect of theory, Shinkarenko et al. [7] obtained the pressure distribution in the fluid film and the elastic deformation of the elastomer by establishing and solving the theoretical model, which provided the best parameters for the design of the surface texture. Hadinata et al. [8] conducted a numerical analysis to study the effect of deterministic microasperities on the elastohydrodynamic performance of lip seal. The micropores on the shaft surface show a significant decrease in friction and an increase in reverse pumping rate.

Most of the current researches focus on the study of flexible friction couples by manufacturing the surface texture on the surface of soft materials, in this way, the texture is easily destroyed during the sliding process, which will seriously affect the life of the friction couple. In this study, surface texture will be manufactured on the surface of rigid bodies, the friction coefficient between the textured rigid sample and smooth flexible sample under dry friction and low sliding speed will be tested by self-made frictional test bench, and the friction mechanism of flexible friction couples will be explored, the results are expected to provide some basis for engineering and living applications.

\section{Experimental setup}

\subsection{Preparation of samples}

The two contact surfaces used in the friction test are made of photosensitive resin and PDMS respectively, where the hardness of the photosensitive resin is much greater than PDMS, which is a rigid body compared to PDMS.

After a series of mechanical analysis, the appropriate surface texture were designed on the rigid body as shown in Fig. 1. Textured resin samples with $\mathrm{R}=0.7, \mathrm{R}=1.1$ and $\mathrm{R}=1.5$ and a smooth resin sample were respectively modeled by software and manufactured by stereolithography (SLA).

\footnotetext{
"Corresponding author's e-mail: zhuxuli@tsinghua.org.cn
} 


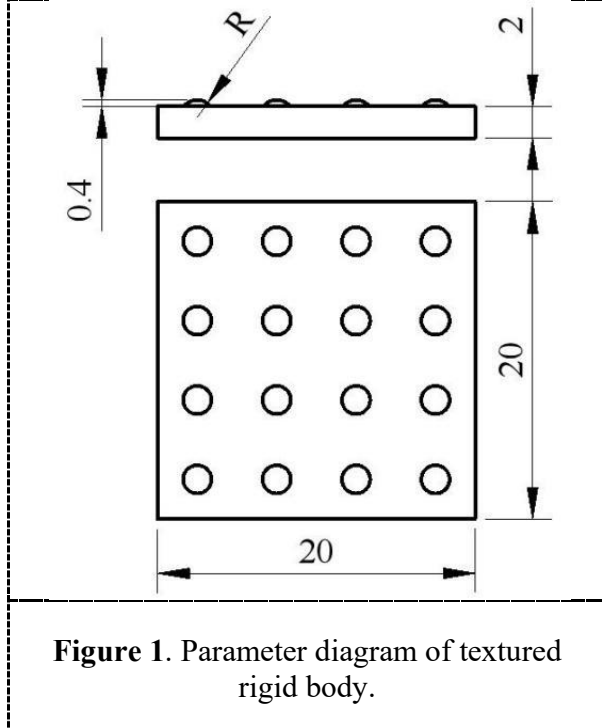

The raw material used to make PDMS samples was SYLGARD 184 Silicone Elastomer. In order to increase the hardness of the samples, a certain amount of silica should be added. The silicone elastomer, catalyst and silica were mixed according to a mass ratio of 30:3:1 and stirred at high speed to make them fully mixed, then the PDMS sample can be obtained by vacuumizing and heating the mixed material. The Shore hardness of the PDMS was measured as 55HA.

\subsection{Frictional test}

The test bench for friction measurement is shown in Fig. 2. The spindle of the stepper motor is connected to the lead screw, the lead screw is meshed with the slide table, and so that, as the lead screw rotates in clock-wise and counter clockwise direction the slide table will move back and forth. The weight is placed on the load box located on the top of the slide table, pressure sensor is attached at the right end of the load box, PDMS is fixed on the top surface of slide table, and resin is fixed on the bottom surface of the load box, the surfaces of the PDMS and the resin facing each other are both selected as the two friction surfaces. The slide table is set to move at a speed rate of $5 \mathrm{~mm} / \mathrm{s}$ by controlling the rotational speed of the motor. When the slide table moves to the right, the load box remains stable, at this point, the positive pressure measured by the sensor is equal to the friction force of the contact surfaces.

The total mass of the load box and the resin is $0.05 \mathrm{~kg}$, a weight of $(0.1 \mathrm{~kg}-1 \mathrm{~kg})$ was added to the load box and the friction experiment was carried out, the total weight of the box and the weight inside was the normal load acting on the friction couple.

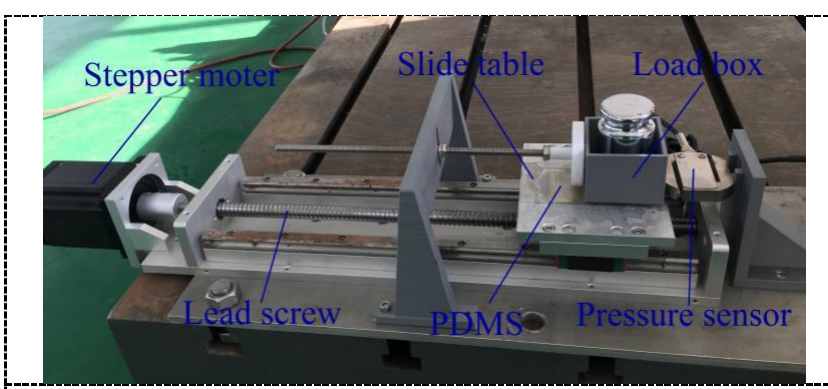

Figure 2. The built test bench for friction experiment.

\section{Results and discussion}

\subsection{Experimental results}

The formula for calculating the equivalent friction coefficient is as follows:

$$
\mu_{\mathrm{e}}=\frac{F_{\mathrm{f}}}{F_{\mathrm{n}}}
$$

where, $F_{\mathrm{f}}$ is the measured friction force $(\mathrm{N})$, and $F_{\mathrm{n}}$ is the normal load $(\mathrm{N})$.

The dependence of the equivalent friction coefficient on the normal load was tested as shown in Fig. 3.

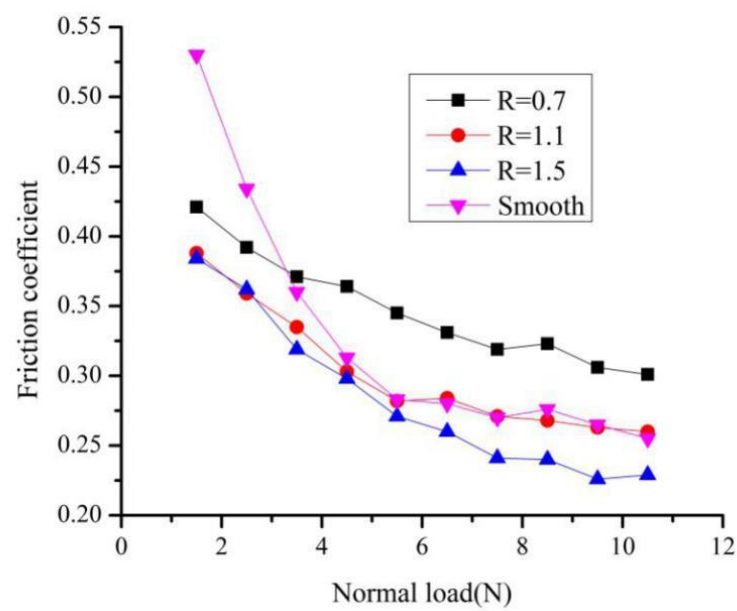

Figure 3. Variations of equivalent friction coefficient of the flexible friction couples with the normal load.

From Figure 3, it can be clearly seen that, the equivalent friction coefficient of both textured and smooth surfaces decreases with the increase of normal load, but the change rate of the smooth surface is larger. Surface texture is capable of reducing friction at low applied normal load compared with the non textured friction couple, as well as increasing friction at high load, in addition, smaller texture radii tend to increase friction.

\subsection{Discussion}

Rubber is a material with high adhesion, friction between smooth contact surfaces is mainly consisting of sliding friction and adhesion. The adhesion comes from the 
force between the molecules on the contact surfaces, which usually increases with the increase of the actual contact area, regardless of the load [9]. The adhesive force accounts for a large proportion in the composition of friction force when the normal load is small, so the friction coefficient is large at this time. However, with the increase of the normal load, the sliding friction increases, and the force caused by adhesion is basically unchanged, so the friction coefficient will be greatly reduced.

The actual contact area of the textured couples is very small, so the effect of adhesion on friction can be ignored. PDMS is relatively soft, so the spherical convex texture will be embedded in the rubber, resulting in large deformation of the rubber. Elastomer will squeeze the texture due to deformation, apparent friction is caused by extrusion and sliding in the contact area.

In order to facilitate the explanation of the friction mechanism, a stress analysis is performed on the texture and the contact model is simplified to two dimensions as shown in Figure 4. where $f_{\mathrm{n}}$ is the normal stress on the texture caused by rubber recovery deformation, $f_{\mathrm{f}}$ is the shear stress due to the sliding of the surfaces, it can be known that $f_{\mathrm{f}}=\mu f_{\mathrm{n}}$, where $\mu$ is the real friction coefficient at this point. According to the principle of mechanical balance, the friction is equal to the sum of the components of $f_{\mathrm{n}}$ and $f_{\mathrm{f}}$ in the horizontal direction:

$$
F_{\mathrm{f}}=\int\left(f_{\mathrm{n}} \sin \alpha+f_{\mathrm{f}} \cos \alpha\right) \mathrm{ds}
$$

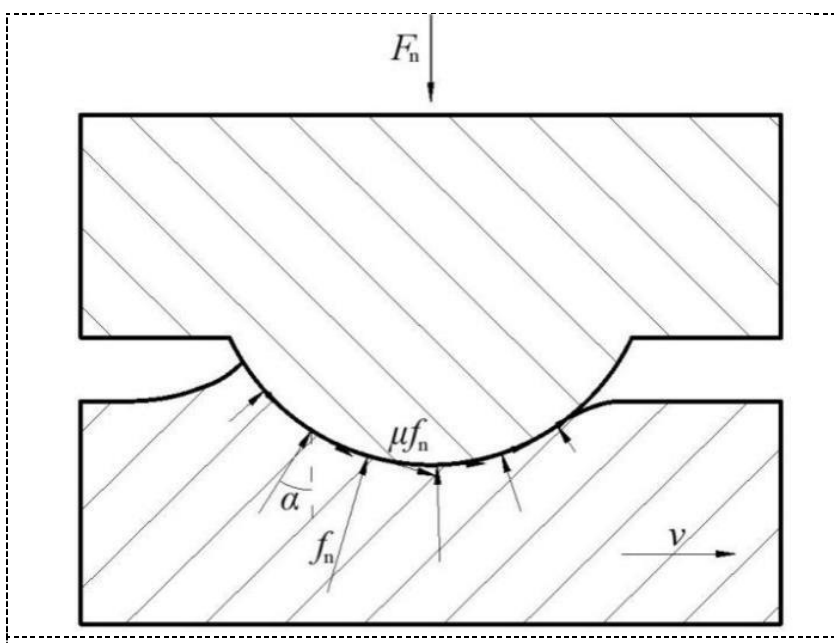

Figure 4. Stress analysis of spherical convex texture embedded in the PDMS.

Since $f_{\mathrm{n}}$ is much larger than $f_{\mathrm{f}}$, it can be seen that the extrusion between the rubber and the texture is the main factor affecting the friction, the force that prevents movement caused by extrusion named ploughing force. It is easy to understand that the deeper the texture is embedded in the rubber, the greater the ploughing force and the greater the apparent friction. Under the same normal load, the friction surface with small texture radius is easier to be embedded into PDMS, which is why the friction coefficient decreases as the texture radius increases.

The rubber with high elasticity will squeeze the texture in the whole contact area, however, the stress on the right half of the texture will restrain the increase of friction, and with the normal load increases, its inhibitory effect becomes more obvious, this will lead to a small increase in the apparent friction, so the friction coefficient decreases accordingly, but it can be seen from the experimental results that the friction enhancement effect of the textured surface is still considerable under the action of large loads.

The above study explored the friction mechanism of flexible friction couples from the mechanical point of view, the research results can provide certain basis for life or engineering application, but the tribology of elastomers is complicated because it can cause large nonlinear deformation during contact, moreover, the surface topography, hardness and adhesion of different kinds of rubber are quite different, so the tribology of rubber still has a great development space.

\section{4 conclusions}

The friction coefficient of flexible friction couples composed of a rigid body (photosensitive resin) with spherical convex texture and a smooth PDMS was tested, the following conclusions can be obtained by comparison with non-textured friction couple:

(1) The equivalent friction coefficient of both textured and smooth surfaces decreases with the increase of positive pressure, but the change rate of the smooth sample is larger.

(2) Spherical convex surface texture is capable of reducing friction at low applied normal load compared with the non-textured friction couple, as well as increasing friction at high load.

(3) The smaller the texture radius, the larger the equivalent friction coefficient of the friction couple.

\section{Acknowledgments}

The work was supported by the National Natural Science Foundation of China (No. 51575323).

\section{References}

1. Gachot C, Rosenkranz A, Hsu S M, et al. (2017) A critical assessment of surface texturing for friction and wear improvement. Wear, 372: 21-41.

2. Etsion I. (2005) State of the art in laser surface texturing. J. Trib., 127(1): 248-253.

3. Abdel-Aal H A. (2016) Functional surfaces for tribological applications: inspiration and design. Surface Topography: Metrology and Properties, 4(4): 043001.

4. Zhang X, Jia J. (2015) Frictional behavior of micro/nanotextured surfaces investigated by atomic force microscope: A review. Surface Review and Letters, 22(06): 1530001.

5. Huang W, Jiang L, Zhou C, et al. (2012) The lubricant retaining effect of micro-dimples on the 
sliding surface of PDMS. Tribology International, 52: 87-93.

6. Li J, Zhou F, Wang X. (2011) Modify the friction between steel ball and PDMS disk under water lubrication by surface texturing. Meccanica, 46(3): 499-507.

7. Shinkarenko A, Kligerman Y, Etsion I. (2009) The effect of surface texturing in soft elastohydrodynamic lubrication. Tribology International, 42(2): 284-292.

8. Hadinata P C, Stephens L S. (2007) Soft elastohydrodynamic analysis of radial lip seals with deterministic microasperities on the shaft. Journal of Tribology, 129(4): 851-859.

9. Wen S, Huang P. (2012) Principles of tribology. Tsinghua University Press, Beijing. 